\title{
SULLA FISSIONE SPONTANEA DELL'URANIO TERRESTRE $\left.\quad{ }^{*}\right)$
}

\author{
C. Festa - M. Santangelo
}

1. - Fin dal 1939, epoca delia scoperta della fissione $\left({ }^{1}\right)$, W. F. Lilhy (-) mise in rilievo la importanza che la eventuale esistenza di un processo di fissione spontanea può assumere nei confronti della geofisica, oltre che della fisica nucleare. Il tentativo compiuto da questo autore di rivelare i neutroni emessi nella fissione spontanea mediante un contatore a pareti di boro non ebhe successo: purtuttavia considerazioni di vario genere lo indussero a stabilire un limite inferiore di ca. $10^{14}$ an per la vita media di questo processo. La possibilità teorica della fissione spontanea dei più pesanti fra i nuclei fu discussa in un fondamentale lavoro da Bohr e Wheeler ( ${ }^{3}$ ), i quali tentarono pure una trattazione quantitativa del problema. Nel 1940 Flerov e Petrzlıak $\left({ }^{4}\right)\left({ }^{5}\right)$ rivelarono i frammenti di fissione con una grossa camera di ionizzazione. In seguito la fissione spontanea dell'uranio fu studiala da numerosi autori $\left({ }^{6}\right)-\left({ }^{10}\right)$; recentemente le caratteristiche del fenomeno per il torio, protoattinio, uranio e per i transuranici sono slate indagate da Segrè ed altri $\left({ }^{11}\right)\left({ }^{12}\right)$ : sono stati così determinati $i$ valori della vita media per fissione spontanea di questi nuclidi.

11 rendimento nel processo di fissione artificiale dell'uranio per ciascuno dei prodotti di fissione è ormai da tempo conosciuto $\left(^{13}\right)$. E noto fra l'altro che tra di essi si trovano alcuni isotopi stabili di gas rari e precisamente del kripton $\left({ }_{36} K r^{83},{ }_{36} K r^{84},{ }_{36} K r^{86}\right)$ e dello xenon $\left({ }_{54} \mathrm{Y} e^{129}, 5_{4} \mathrm{X} e^{131},{ }_{51} \mathrm{X}^{134},{ }_{-4} \mathrm{Y}^{136}\right)$. L'andamento dello yeld della fissione spontanea per ciascuno di questi nuclidi è stato determinato da MacNamara c Thode $\left({ }^{14}\right)$, i quali hanno esaminato la composizione isotopica del kripton e dello xenon estratti dalla pecblenda. Dal confronto fra le abbondanze relative degli isotopi di fissione e quelli non di fissione del kripton e dello xenon, essi hanno desunto la prova della

(\$) Comunicazione presentata al Convegno dell Issociazione Ceofisica Italiana, tenuto a Roma il 10-11 giugno 1952. 
presenza di prodotti di fissione nei gas estratti dalla pecblenda e, precisamente, dei tre isotopi del kripton e dei cinque dello xenon, menzionati poc'anzi. Hanno inoltre riscontrato la presenza di gas atmosferici nella pechlenda; questo si deduce dal fatto che le abbondanze relative degli isotopi non provenienti dalla fissione sono identiche a quelle atmosferiche. Partendo da queste considerazioni, i due autori hanno potuto calcolare lo yeld della fissione spontanea per le masse: $83,84,86$, e 129, 131, 132, 134, 136 ed hanno trovato che esso i, salvo alcuni spostamenti, analogo a quello della fissione indotta.

2. - Lo studio dei gas rari ha un grande interesse per le informazioni che dai risultati di esso si possono trarre riguardo al meccanismo di formazione dell'atmosfera e della crosta terrestre. Com'è noto delti elementi non sono influenzati dalle reazioni chimiche, sicché la loro storia va sempre interpretata in funzione dei fenomeni fisici che possono avere alterata l'abbondanza dell'elemento e deg̣li isotopi dei quali esso si compone. Fra questi fenomeni vanno poste in primo piano le disintegrazioni nucleari che possono causare variazioni locali delle abbondanze di entità non trascurabile. L'alterazione della composizione isotopica dell'argon terrestre $\left({ }^{15}\right)$ e la anomala al)bondanza di questo gas nell'atmosfera, dovute al decadimento del $K^{\text {"l }}$ per cattura elettronica, costituiscono ormai un esempio classico di simili variazioni. Altri fenomeni del genere sono stati osservati e nel nostro pianeta e nelle atmosfere stellari $\left({ }^{16}\right)$. Un problema hasilare per la interpretazione delle regole di abbondanza degrli elementi nel cosmo e sulla terra è appunto quello di conoscere la entità dell'influsso esercitato da simili processi sulle abbondanze originarie dei was rari. Per es., H. Brown ( ${ }^{3 i}$ ) ha calcolato le abbondanze cosmiche di questi gas, fondandosi sulla ipotesi che lo xenon e il kripton, presenti nell'atmosfera terrestre, non siano stati alterati nel tempo da alcun processo nucleare.

Poiché, come abbiamo visto poc'anzi, fra i nuclidi stabili prodotti dalla fissione spontanea si trova un buon numero di isotopi di questi due gas, il presente lavoro è stato intrapreso con lo scopo di indagare se il fenomeno della fissione spontanea dell'uranio abbia prodotto un'alterazione sensibile della loro abbondanza originaria. Si può peraltro osservare a priori che lo yeld della fissione per i singoli isotopi del kripton e dello xenon raggiunge il suo valore massimo in corrispondenza delle masse 86 e, rispettivamente, 136 , mentre invece il $K r^{86}$ e lo $X e^{136}$ non sono fra gli isotopi più abbondanti del kripton e dello 
xenon atmosferici. Questo potrebbe significare ehe il processo originario di formazione del kripton e dello xenon che si riscontrano nell'atmosfera ha avuto un carattere diverso da quello della fissione. In altre parole la fissione non dovrebbe avere influito in maniera sensibile sulla formazione dei gas rari dell'atmosfera terrestre.

3. - La quantità di kripton e xenon generata dall'uranio terrestre a partire dall'epoca di formazione della terra è legata al contenuto altuale di uranio nella terra; perciò si è tentato come prima cosa una valutazione approssimativa di tale contenuto.

Per quanto riguarda il contenuto di uranio nella crosta terrestre esistono misure eseguite direttamente sulle rocce che la compongono: si tratta però, nella massima parte dei casi, di misure del contenuto percentuale di radio dalle quali si risale al contenuto di uranio attraverso la nota relazione:

$$
U / R a=2,8 \times 10^{6}
$$

valida per l'equilibrio radioattivo. Sarà bene avvertire fin da ora che l'esistenza di questo equilibrio non può asserirsi con sicurezza per ogni tipo di roccia $\left({ }^{18}\right)$.

Il contenuto di uranio nei sedimenti marini $\left({ }^{19}\right)$ è $\mathrm{di} 1,07 \times 10^{6} \mathrm{~g} / \mathrm{g}$ e nei rimanenti sedimenti $\left({ }^{20}\right)$ è di $1,25 \times 10^{-6} \mathrm{~g} / \mathrm{g}$. Se si tiene conto del fatto che il $70,8 \%$ dei sedimenti è costituito da sedimenti marini ( $\left.{ }^{-1}\right)$, il contenuto medio di uranio nello strato dei sedimenti risulta di $1,12 \times 10^{-6}$ gr gr. Assumendo con Gutenherg (22) per lo spessore medio dello strato dei sedimenti il valore di $5 \mathrm{~km}$ e per la sua densità ( $\left.{ }^{23}\right) 2,3 \mathrm{~g}^{2} \mathrm{~cm}^{-3}$, si può calcolare che in questo strato sono presenti $7,9 \times 10^{21}$ g di uranio. Lo strato del granilo si compone $\left({ }^{24}\right)$ per il $52,5 \%$ di graniti, per il $39,3 \%$ di rocce ignee intermedie e per $19.2 \%$ di roce basico-ignce. Poiché il contenuto di uranio $\left({ }^{25}\right)$ nei graniti $\grave{c}: 3,96 \times 10^{-6} \mathrm{a} / \mathrm{g}$, nelle roce ignee-intermedie $2,61 \times 10^{-6}$ $\underline{g}^{\prime} \stackrel{\text { r }}{\mathrm{C}}$ e nelle roce basico-imnee: $0,96 \times 10^{6} \mathrm{~g} / \mathrm{g}$, il contenuto medio di uranio nello strato del granito risulta pari a ca. $3,18 \times 10^{-6} \mathrm{~g} / \mathrm{g}$. Se si prende come spessore medio continentale (20) dello strato del granito $20 \mathrm{~km}$ e come spessore medio oceanico $\left({ }^{23}\right) 10 \mathrm{~km}$, tenendo conto del fatto che le aree continentali $\left(^{26}\right)$ costituiscono il $29,2 \%$ della superficie terrestre $\left.{ }^{2 \pi}\right)$, si può porre lo spessore medio del granito pari a $13 \mathrm{~km}$. Allora, dato che la densita dei graniti ( $\left.{ }^{23}\right)$ vale circa 2,65 g $\mathrm{cm}^{-3}$, si può calcclare ele l'uranio centenuto nello strato 
del granito ammonti a $5,9 \times 10^{13} \mathrm{~g}$. Considerazioni analoghe a quelle seguite per la valutazione dello spessore dello strato del granito possono applicarsi al calcolo dello spessore dello strato intermedio, che risulta dell'ordine di $16 \mathrm{~km}$.

Come contenuto di uranio nello strato intermedio si può assumere il contenuto di uranio nei basalti $\left({ }^{30}\right)$ e cioè : $0,83 \times 10^{-6} \mathrm{~g} / \mathrm{g}$. Con questi due valori, tenuto conto del valore della densità dello strato intermedio $\left.{ }^{23}\right)\left(2,87 \mathrm{~g} \mathrm{~cm}^{-3}\right)$, si trova che nello strato intermedio sono presenti $1,9 \times 10^{19} \mathrm{~g}$ di uranio. I dati relativi al contenuto di uranio nei singoli strati della crosta terrestre, adoperati nelle precedenti considerazioni concordano in modo soddisfacente con $i$ dati relativi al contenuto di radio negli stessi strati, riportati da Gutenberg (22).

Una valutazione del contenuto di uranio nelle parti più interne della terra non può ottenersi senza formulare ipotesi, finora praticamente incontrollabili, sulla composizione chimica dell'interno della Terra. Attualmente le ipotesi più accettate riguardo alla natura del mantello sono: 1) la composizione chimica del mantello è simile a quella delle duniti $\left({ }^{28}\right)$; 2) dalla base della crosta fino a $1000 \mathrm{~km} \mathrm{la}$ composizione chimica del mantello è analoga a quella delle aeroliti, da $1000 \mathrm{~km}$ fino alla somità del nucleo è analoga a quella delle sideroliti. Nella prima ipotesi si avrebbe nel mantello un contenuto di uranio $\left({ }^{30}\right)$ pari a $0,013 \times 10^{-6} \mathrm{~g} / \mathrm{g}$. Se fosse vera la seconda il contenuto di uranio $\left({ }^{31}\right)$ sarebbe di $0,029 \times 10^{-6} \mathrm{~g} / \mathrm{g}$ fino a $1000 \mathrm{~km}$ e di $0,012 \times 10^{-6} \mathrm{~g} / \mathrm{g}$ da $1000 \mathrm{~km}$ alla base del mantello. Con i dati relativi alla distribuzione delle densità nell'interno del mantello ottenuti da Bullen $\left({ }^{23}\right)$ si può calcolare che l'uranio contenuto nel mantello sia nell'ordine di $5,5 \times 10^{19} \mathrm{~g}$ nella prima ipotesi e di $5,3 \times 10^{19} \mathrm{~g}$ nella seconda. L'adozione dell'una o dell'altra ipotesi non ha quindi importanza per $\mathrm{i}$ fini che si propone la presente ricerca.

La composizione chimica del nucleo terrestre è generalmente ritenuta simile a quella delle sideriti $\left({ }^{24}\right)\left({ }^{29}\right)$; esistono però altre teorie sulla natura del nucleo: la teoria di Kuhn e Rittmann $\left({ }^{32}\right)$ secondo la quale il nucleo sarebbe costituito di materia solare indifferenziata, ossia praticamente di idrogeno; la teoria di Ramsey ( $\left.{ }^{23}\right)$, clee considera il mantello ed il nucleo come fasi termodinamiche differenti di una identica sostanza. Scartata in un primo tempo la ipotesi di Kulın e Rittmann, che è notoriamente la più discussa e che comunque escluderebbe la presenza di uranio nel nucleo, nella prima ipotesi (sideriti) il contenuto di uranio nel nucleo $\left({ }^{31}\right)$ risulterebbe pari a 
$0,003 \times 10^{-6} \mathrm{~g} / \mathrm{g}$, mentre nella ipotesi di Ramsay sarebbe uguale a $\left({ }^{30}\right)$ $0,013 \times 10^{-6} \mathrm{~g} / \mathrm{g}$. Di conseguenza considerando che la massa del nucleo, secondo i dati ogggi più attendibili $\left({ }^{23}\right)$, è compresa fra 1,85 e $1,89 \times 10^{27} \mathrm{~g}$, si ottiene un contenuto di uranio nel nucleo di $0,5 \times 10^{10} \mathrm{~g}$ prima ipotesi e di $2,4 \times 10^{10} \mathrm{~g}$ nel caso che sia verificata la ipotesi di Ramsey.

In quest'ultima ipotesi i dati relativi all'uranio presente nella terra sono quelli raccolti nella Tabella $I$ : essi corrispondono ad un contenuto totale di $1,65 \times 10^{20} \mathrm{~g},\left(1,4 \times 10^{20} \mathrm{~g}\right.$ nell'ipotesi di Kuhn e Rittmann).

TABFLla I

\begin{tabular}{|c|c|c|c|}
\hline & Crosta & Mantello & Nucleo \\
\cline { 1 - 2 }$\times \begin{array}{c}11 \\
\times 10^{10} \mathrm{~g}\end{array}$ & 8,6 & 5,5 & 2,4 \\
\hline $\begin{array}{c}\mathrm{C}^{234} \\
\times 10^{41.1 t o m i}\end{array}$ & 2,2 & 1,4 & 0,6 \\
\hline
\end{tabular}

Gli isotopi naturali dell'uranio attualmente conosciuti sono tre: $U^{234}, U^{-35}$ e $U^{235}$. Un confronto fra le abbondanze di questi nuclidi e i valori delle vite medie per emissione $\alpha$ e per fissione spontanea di ciascuno di essi mostra come nei problemi di geofisica può assumere una certa importanza unicamente la fissione spontanea del nuclide di massa 238. Tenuto conto dell'abbondanza di questo isotopo $\left({ }^{3 \cdot i}\right)$ si ha che nella terra sono presenti $4,2 \times 10^{41}$ atomi di $U^{238}$, distribuiti conformemente alla seconda riga della Tabella $I$.

Ci si potrebbe domandare a questo punto se sia lecito estendere all'intera terra i valori delle abbondanze isotopiche ${ }^{34}$ ), misurate su campioni provenienti dalla parte più superficiale di essa. Sta di fatto che i dati - sia pure relativamente scarsi - riguardanti le abbondanze isotopiche nelle meteoriti e nelle atmosfere stellari, provano che, salvo alcune eccezioni, tali ablondanze si mantengono costanti nell'universo ( $\left.{ }^{35}\right)$.

4. - 11 numero di nuclei di massa $A$, prodotti nel tempo $t$ dall' $U^{235}$ per fissione spontanea è dato da:

$$
N_{\mathrm{A}}=\frac{\lambda_{\mathrm{f}}}{\lambda_{\alpha}} \frac{Y_{\mathrm{A}}}{100} N_{\mathrm{s} 3 \mathrm{~s}}\left(e^{\lambda_{t}}-1\right)
$$


dove $i_{f}$ è la costante di decadimento per fissione spontanea $\left(8,6 \times 10^{-17} \mathrm{an}^{-1}\right)$, $\lambda_{\alpha}$ la costante di decadimento per emissione $"$ $\left(1,5 \times 10^{-10}\right.$ an $\left.^{-1}\right)$ e $\lambda=\iota_{f}+\lambda_{\alpha}$ è la costante di decadimento totale (praticamente uguale a $\left.\lambda_{i}\right) ; Y_{A}$ è lo yeld della fissione spontanea relativo alla massa $A$. Se si pone $t=3,3 \times 10^{9}$ an, valore comunemente accettato per l'età della terra $\left({ }^{30}\right)$, e si attribuiscono ad $N$ \%, successivamente, i singoli valori riportati nella seconda riga della Tabella $I$, si può calcolare il numero di nuclidi di massa $A$ generati dall'Uranio nei singoli strati della terra dall'epoca di formazione di questa. 11 calcolo è stato eseguito per le masse 83, 84, 86, 129, 131, 132, 134, 136. I risultati sono raccolti nella Tabella II: da essi si desume che in $3,3 \times 10^{9}$ an, per la fissione spontanea dell' $U^{23 s}$ contenuto nell'intera terra, si sono formati $3,5 \times 10^{33}$ atomi di kripton e $2,5 \times 10^{34}$ atomi di xenon, pari rispettivamente al $0,004 \%$ del kripton e al $0,3 \%$ dello xenon atmosferici. Un risultato simile a questo sembra abbiano ottenuto Khlopin e Gerling $\left({ }^{37}\right)$ in una ricerca sul kripton e lo xenon prodotti dall'uranio terrestre. Sicché, tutto il kripton e lo xenon, prodotti dall'uranio terrestre per fissione spontanea costituirebbero una frazione molto piccola dei corrispondenti gas atmosferici.

\section{TABELLA II}

\begin{tabular}{|c|c|c|c|}
\hline \multirow{2}{*}{ A } & \multicolumn{3}{|c|}{$\begin{array}{l}\text { N di atomi di massa } A \text { prodotti in } 3,3 \times 10^{9} \text { an per fissione } \\
\text { spontanea dell'U } 238 \text { contenuto in }\end{array}$} \\
\hline & $\begin{array}{c}\text { Crosta } \\
\text { at } \times 10^{33}\end{array}$ & $\begin{array}{l}\text { Mantello } \\
\text { at } \times 10^{32}\end{array}$ & $\begin{array}{l}\text { Nucleo } \\
\text { at } \times 10^{32}\end{array}$ \\
\hline 83 & 1,0 & 0,6 & 0,3 \\
\hline 81 & 3,7 & 2,4 & 1,0 \\
\hline 86 & 13,5 & 8,6 & 3,5 \\
\hline 129 & 0,6 & 0,4 & 0,2 \\
\hline 131 & 6,1 & 3,9 & 1,6 \\
\hline 132 & 28,6 & 18,3 & 7,4 \\
\hline 134 & 42,1 & 26,9 & 11,0 \\
\hline 136 & 49,6 & 31,7 & 23,9 \\
\hline
\end{tabular}

Pù̀ avere un certo interesse il confronto delle abbondanze dei singoli isotopi del kripton e dello xenon atmosferici con le abbondanze dei singoli nuclidi generati dall'uranio terrestre. Questi dati sono riportati nella Tabella III dove con $N$ a $^{\text {a }}$ indica il numero di atomi di massa $A$ presenti nell'atmosfera e $N_{A}{ }^{\mathrm{f}}$ i corrispondenti prodotti di fissione. 
5. - Il confronto fra il kripton e lo xenon originati dalla fissione ed il contenuto degli stessi elementi nell'interno della terra avrebbe una importanza di gran lunga superiore a quello precedentemente istituito con il kripton e lo xenon atmosferici. Esso servirel,be a stabilire da un lato il contributo della fissione spontanea alla formazione del kripton e dello xenon contenuti nell'interno della terra e l'influsso da essa esercitato sulla loro composizione isotopica, e dall'altro le relazioni fra questi gas e i corrispondenti nell'atmosfera, le quali interessano il problema del meccanismo di formazione dell'atmosfera stessa $\left(^{15}\right)$. Purtroppo attualmente un simile confronto è irrealizzabile per la mancanza di dati sperimentali attendibili sul contenuto di kripton e xenon nelle rocce.

Tabella III

\begin{tabular}{|c|c|c|c|c|c|c|c|c|}
\hline $\mathbf{A}$ & 83 & 84 & 86 & 129 & I3I & $\mathrm{I} 32$ & 134 & 136 \\
\hline $\begin{array}{l}\mathrm{N}_{\mathrm{A}} \mathrm{e} \\
\text { at } \times 10^{33}\end{array}$ & 0,2 & 0,7 & 2,6 & $0, \mathrm{I}$ & $\mathrm{I}, 2$ & 5,4 & 8 & 10 \\
\hline $\begin{array}{l}\mathrm{N}_{\mathrm{A}}^{\mathrm{a}} \\
\text { at } \times 10^{36}\end{array}$ & 12,5 & 61,3 & 18,3 & 2,2 & 1,8 & 2,3 & 0,9 & 0,7 \\
\hline
\end{tabular}

Una valutazione di tale contenuto è stata tentata da Brown (17): si tralla in verita di una estrapolazione fondata su alcune misure di Cady e Cady $\left({ }^{38}\right)$. Questi ultimi banno determinato la quantità di $\boldsymbol{K} \boldsymbol{r}+\boldsymbol{X} e$ presente in alcuni gas naturali, ottenendo valori che scartano notevolmente l'uno rispetto all'altro. Non si sa, quindi, quanto sia lecito assumere la media di questi valori come il contenuto di $\boldsymbol{K} \boldsymbol{r}+\boldsymbol{X} \boldsymbol{e}$ in tutti $\mathrm{i}$ gas naturali. Comunque, dai dati riportati nella Tabella V del citato lavoro di Brown, seguendo il metodo sviluppato dal Brown stesso, si ricava che nella crosta terrestre si troverebbero $5,3 \times 10^{35}$ atomi di kripton e $4,2 \times 10^{34}$ atomi di xenon. Se questo fosse vero il kripton e lo xenon, prodotti in $3,3 \times 10^{9}$ an per fissione spontanea dell'uranio contenuto nella crosta, risulterebbero pari rispettivamente al $0,3 \%$ del kripton e al $30 \%$ dello xenon, contenuti nella crosta medesima: il contributo della fissione spontanea non sareblse in tal caso trascurabile. E ovvio cbe queste valutazioni, la cui incertezza è già stata rilevata, hanno un valore puramente indicativo: servono tuttavia a dimostrare quale interesse avrebbe la determina- 
zione delle abbondanze e della composizione isotopica del kripton e dello xenon nelle rocce e nei gas naturali.

Roma - Istituto Nazionale di Geofisica - Agosto 1952.

\section{RIASSUNTO}

Partendo dai dati più recenti sulla vita media della fissione spontanea delluranio, gli A. tentano una valutazione delle quantita di $\mathrm{Kr}$ e Xe generate in questo processo dall'uranio contenuto nella terra a partire dall'epoca di formazione della crosta terrestre. Il confronto delle abbondanze dei singoli isotopi dei due elementi contenuti nell'atmosfera con le abbondanze degli stessi isotopi generati per fissione, porta a concludere che il contenuto della fissione spontanea dell' $\mathrm{U}^{23 s}$ alla formazione del $\mathrm{Kr}$ e $\mathrm{Xe}$ atmosferici è trascurabile.

Dal confronto tra le quantità di $\mathrm{Kr}$ e Xe di fissione e il contenuto degli stessi elementi nella crosta terrestre, calcolato secondo Brown, risulterebbe invece che in tal caso la fissione spontanea delluranio non sarebbe da trascurare.

Si prospetta infine, a conclusione, linteresse di eseguire determinazioni delle abbondanze e della composizione isotopica del $\mathrm{Kr}$ e $\mathrm{Xe}$ nelle rocce e nei gas naturali.

\section{SUMMARY}

A rough determination of the $\mathrm{Kr}$ and $\mathrm{Xe}$ generated by the spontaneous fission of the terrestrial uranium during the last 3,3 billion years (earth's age) is attempted in this worl. The calculations have been based on the most recent data for the spontaneous fission mean life of $\mathrm{U}^{238}$. From a comparison between the abundances of each isotope in the atmospherical $\mathrm{Kr}$ and $\mathrm{Xe}$ and the abundances of the same isotope in the fission $\mathrm{Kr}$ and $\mathrm{Xe}$, one can estimate that the contribution of the spontaneous fission of $\mathrm{U}^{238}$ to the production of the atmospherical $\mathrm{Kr}$ and $\mathrm{Xe}$ is negligeable. The fission $\mathrm{Kr}$ and $\mathrm{Xe}$ and the $\mathrm{Kr}$ and $\mathrm{Xe}$ content in the earth's crust (calculated following the Brown's method) are also compared. The spontaneous fission of U seems not negligeable in this case.

The great interest of the determination of the $\mathrm{Kr}$ and $\mathrm{Xe}$ content in rocks and natural gases and its isotopic composition is prospected. 


\section{BIBLIOGRAFIA}

(1) Hahn O. und Stinssmane F.: Naturw. 27, 11 (1939).

(-) Liber W. F.: Pliys. Rev, 55, 1269 (1939).

(3) Bohl N. and Wheeler J. A.: Phys. Rev., 56, 426 (1939).

(4) Fleiov G. N. and Petrzhak K. A.: Journ. Phys. U.S.S.R., 3, 275 (1940). Chem. Abstr. 35, 3677 (19.11).

(5) Fleiov G. N. and Petrzhak K. A.: Pliys. Rev, 58, 89 (1940).

(G) Maurer W. und Pose H.: Zs. Phys, 121, 285 (19.13).

(7) Pose H.: Zs. Plyys., 121, 293 (19.13).

(8) Scharff.Goldhareir G. ind Klaiber S. G.: Plyys. Rev., 70, 229 (19.16).

(9) Penfilov N. A.: Journ. Pliys. U.S.S.R, 11, n. 3 (1947), Chenı. Abstr. 42, 286.tb (19.18).

(10) Yacoda H. and Karlan N.: Pliys. Rev, 76, 702 (1949).

(11) Segrè E.: AEC Decl. Doc., LADC.975 (May 8, 1951), Phys. Rev. 86, 21 (1952).

(12) Seabong; G. T.: Phys. Rev., 85. 158 (1952).

(13) Plutonium Project: Rev. Mod. Plyys., 18, 512 (19.16).

(1.) Mac-Namara J. and Tuode H. G.: Phys. Rev., 80, 171 (1950).

(15) Bonto G., Canem G. e Santangelo M.: Nuovo Cimento 9, H (1952).

(16) Ganow G. and Cututinfield C. L.: Theory of Atomic Nucleus and Atomic Energy Sources. Oxford (1950).

(1i) Brown H.: in The Atmosphere of the Earth and Planets. ed. G. KuiperChicago (19-19) pag. 260-268.

(18) Bacheltet F., Fenretti Sforzssi M. e Ippolito F.: Nuovo Cimento; 8, 851 (1951).

(19) Uniry W. D.: Am, Journ. Sci., 239, 191 (1911).

(20) Evans R. D. and Goobmax C.: Bull. Geol. Am., 52, 459 (19.11).

(21) Kuenen Ph. H.: Marine Geology. New York (1950).

(22) Gutenderc, B.: The Internal Constitution of the Earth, New York (1951).

(23) Butuex K. E.: An Introduction to the Theory of Seismology. Cambridge $(19.17)$.

(-4) DaLy R.: lgneous Rocks and the Depth of the Earth. New York (1933).

(25) Sexftle F. E. and Keevil N. B.: Trans. Am. Geophys. Un. 28, 732 (1947).

(20) Kalle K.: Der Stoffhaushatt des Meeres. Prohlems der Kosnı. Phys. Leipzig (19\3).

(2T) Birci F., Schinarer F. and Spicer H. C.: Hand, of Phys. Const. Geol. Soc. Anı. Pap. 36 (19:12).

(28) Binci F.: J. Geoph. Res. 56, 379 (1951).

(29) Unex H. C.: Geoch. and Cosm. Acta. 1. 209 (1951).

(30) Divis G. L.: Anı. Journ. Sci. 2.15, 677 (1947).

(31) Davis G. L.:Am. Journ. Sci, 248, 107 (1950).

(32) Kuin W. und Ritruas A.: Geol. Rundschau. 32, 215 (19.41).

(33) Rinsey W. H.: M.N.R.A.S. Geoph. Suppl, 5, 109 (19.19).

(34) Ges. El. Res. Lab.: Chart of Nuclides (1950).

(3ii) Brows H.: Rev. Mod. Pliys., 21, 625 (19.19). 
(36) Alpher R. A. and Herman R. C.: Phys. Rev, 84, 1111 (1951).

(37) Khlopin V. G. and Gerlinc E. K.: Radium Inst. Ac. Sci, U.S.S.R. (1948). 6p; Chem. Abstr., 43, 527 (1949).

(38) Cady G. H. and Cady H. P.: Ind, Eng. Chem. Anal. Ed, 17, 760 (1945).

(39) Seaborc G. T. and Perlmann M. L.: Journ. Am. Chem. Soc, 70, 1571 (1948). 